\title{
An Extended Compound Gamma Model and Application to Composite Fading Channels
}

\author{
T. Princy ${ }^{1}, 2$ \\ ${ }^{1}$ Centre for Mathematical Sciences, Arunapuram, Kerala, India \\ ${ }^{2}$ Department of Statistics, Banaras Hindu University, Varanasi, India
}

Received: 15 July 2014; Accepted: 5 February 2015

Editor: David G. Yu

\begin{abstract}
Wireless communication systems are subject to short and long-term fading channels. In this paper, an extended form of a statistical model for the composite fading channels is derived from the maximum entropy principle. Subsequently, the composite fading channel is derived by replacing the conditional density by entropy-maximizing distribution (Mathai's pathway model). This pathway model is versatile enough to represent short-term fading as well as the shadowing. The new wireless channel model generalizes the commonly used models for multipath fading and shadowing. In particular, using the $G$-function, we derive the density function, distribution function and moments of the new model in closed form. These derived results are a suitable device to analyze the performance of composite fading systems such as density function of the Signal Noise to Ratio (SNR), Amount of Fading (AF), and Outage Probability (OP) etc. The results will be shown graphically for different signal and fading parameter values.
\end{abstract}

Keywords Mellin transform, signal to noise ratio, $G$-function, heavy-tailed distribution, Whittaker function, Nakagami distribution.

AMS 2010 subject classifications 33C60, 44A15, 60E05.

DOI: $10.19139 /$ soic.v3i1.91

\section{Introduction}

The modeling of wireless communication channel is one of the fastest growing segments in the communication industry. The applications of wireless system include internet access, web browsing, file transfer, video transfer, entertainment, etc. Recently, various types of statistical models are used for describing the characteristics of wireless communication systems. There are many approaches and several combinations are available in the literature for modeling the composite fading channels. Modeling of composite fading channels is essential for the analysis of several wireless communication problems, including interference analysis in cellular systems and performance analysis of network MIMO, distributed antenna systems and cooperative relay networks. Most of the available channels are based on the superpositions of multipath components. For a brief review of the composite distribution in the context of the wireless communication channel, see [1].

In wireless communication systems, the received signal is likely to suffer from the damaging effects of the channel in the form of short and long-term fading, also known as shadowing, while short term fading arises from the existence of multiple paths between transmitter and receiver, shadowing is the result of the topographical

\footnotetext{
${ }^{*}$ Correspondence to: Princy (Email: princycms@ gmail.com). Centre for Mathematical Sciences, Arunapuram P.O., Palai- 686574, Kerala,
} India.

ISSN 2310-5070 (online) ISSN 2311-004X (print)

Copyright (C) 2015 International Academic Press 
elements such as buildings, trees and other structures in the transmission path. Since both short and long-term fading conditions coexist in wireless systems, the density function of the envelope in a shadowed short-term fading channel becomes

$$
f(x)=\int_{0}^{\infty} h(x \mid y) g(y) \mathrm{d} y .
$$

Many compound models are available in the literature for modeling fading channels, for example, Weibulllognormal [2], Nakagami-lognormal [3], Rayleigh-lognormal [4], Weibull-gamma [5], gamma-lognormal [1], Kdistribution [6], generalized K-distribution [7] etc. Due to the great importance of compound models, Nadarajah and Kotz [8] generated a collection of compound distributions for the statistical modeling of shadowed fading channels. However, the composite fading models are very difficult to handle analytically so, Atapattu et al. [9] used the gamma mixtures for the composite fading channel. The performance analysis in a wireless channel with multiple interferers, subject to shadowing and fading, using a compound model was studied by Shankar [10].

The objective of the present paper is to derive a compound gamma model for the composite fading channels on the basis of the maximum entropy principle. This paper starts with a brief description of the composite fading model. This article is structured as follows: Section 2 discusses the importance of entropy in wireless fading channels. The connection between entropy and pathway model is mentioned in section 3 . In section 4 , we derive an extended compound gamma model in the presence of pathway model. The performance measures such as Amount of Fading (AF), Outage Probability (OP) and Average Bit Error Rate Probability (ABEP) of these systems are obtained in section 5 .

\subsection{Composite fading channel}

Let us start with the construction of composite fading model in wireless channels. Wireless channels are subject to random fluctuations in received power arising from multipath propagation. Often these channels suffer from long-term fading or shadowing arising from multiple scattering conditions. Since shadowing and fading occur simultaneously, various models have been used to describe shadowed fading channels. The density function of the received power $\gamma$, conditioned on the average power $y$, is a Nakagami density of the form

$$
p_{1}(x \mid y)=\frac{2}{\Gamma(m)}\left(\frac{m}{y}\right)^{m} x^{2 m-1} \mathrm{e}^{-\frac{m x^{2}}{y}}, x \geq 0, m, y>0,
$$

and $p_{1}(x \mid y)=0$ elsewhere, where $\Gamma($.$) is the gamma function and m$ is the multipath fading parameter. Note that for $m=\frac{1}{2}$, the density function is half-Gaussian, while for $m=1$, the density function is a Rayleigh. When multipath fading is superimposed on shadowing, the average power $y$ slowly varies and hence it can be considered as a random variable. The fluctuation of the average power due to shadowing is usually modeled by the lognormal distribution. However, in this paper, we use the gamma density for modeling the variation of the average power since it is shown to be a good fit to experimental data [6],[7] and can approximate the lognormal distribution. The form of the model is given by

$$
p_{2}(y)=\frac{1}{\Gamma(\delta)}\left(\frac{\delta}{\beta}\right)^{\delta} y^{\delta-1} \mathrm{e}^{-\frac{\delta y}{\beta}}, y \geq 0, \delta, \beta>0,
$$

and $p_{2}(y)=0$ elsewhere, where $\delta$ is the shadowing parameter and $\beta$ is a measure of the average power. Using (2), (3), (1) and the definition of Bessel function, the form of the density function of the received signal power $\gamma$ is as follows,

$$
\left.p_{\gamma}(x)=\frac{4}{\Gamma(m) \Gamma(\delta)}\left(\frac{m \delta}{\beta}\right)^{\frac{m+\delta}{2}} x^{m+\delta-1} K_{\delta-m}\left(2 \sqrt{(} \frac{\delta m}{\beta}\right) x\right),
$$

for $x \geq 0, \beta, m, \delta>0$ and $p_{\gamma}(x)=0$ elsewhere. 


\section{Entropy in channel modeling}

The principle of maximum entropy provides a theoretical justification to make a model for wireless communication systems out of the information available. The logic behind entropy maximization, popularized by Jaynes [11], is that the Shannon entropy is a good measure of uncertainty, and therefore entropy maximization brings the powerful model given the stated constraints. The role of entropy maximization method as a flexible tool for modeling the correlated wireless channel was first introduced by Debbah and Müller [12]. Guillaud et al. [13] and Debbah and Müller [12] used the concept of entropy maximization for obtaining models for MIMO wireless channels.

The wireless channel suffers from constructive/destructive interference signaling and therefore yields a randomized channel frequency response. The correlation is a critical parameter for communication systems and therefore we will pay special attention to it. Let $p_{H \mid E}$ describe the channel statistics corresponding to a given correlation situation (given the location of the transmitter and receiver arrays and a given set of scatters) and $p_{E}$ denote the model, which depends on the number, position and properties of the antennas in the transmit and receive arrays, as well as of the scatters. Then the complete distribution of the correlated channel $H$ is obtained by marginalizing over $E$ :

$$
p_{H}(h)=\int_{E} p_{H \mid E}(h, E) p_{E}(E) \mathrm{d} E,
$$

and this is the logic behind this method, exposed in more detail in [13]. Our method can be summarized as follows:

1. derive $p_{E}(E)$ through entropy maximization,

2. derive $P_{H \mid E}(H, E)$ through entropy maximization,

3. marginalize over $E$ to obtain

$$
p_{H}(h)=\int_{E} p_{H \mid E}(H, E) p_{E}(E) \mathrm{d} E .
$$

Taking the statistical model with greatest entropy avoids the arbitrary introduction or assumption of information that is not available. From the literature, we can see that entropy maximization method is a fundamental tool for modeling the wireless communication systems. Motivated by the importance of entropy optimization procedure in wireless communication systems, we propose a class of an extended compound gamma model.

\section{Entropy and modeling}

In physical situations when an appropriate model is selected, one procedure is the maximization of entropy. Mathai and Haubold [14] introduced a new entropy measure which is a generalization of the Shannon entropy measure. Consider a multinomial population $p=\left(p_{1}, p_{2}, \ldots, p_{k}\right), p_{i}>0, i=1,2, \ldots, k, p_{1}+p_{2}+\cdots+p_{k}=1$, Mathai entropy measure is given by the relation

$$
M_{k, q}(p)=\frac{\sum_{i=1}^{k} p_{i}{ }^{2-q}-1}{q-1}, q \neq 1, q<2,
$$

and for the continuous case it is

$$
M_{k, q}(f)=\frac{\int_{-\infty}^{\infty}[f(x)]^{2-q} \mathrm{~d} x-1}{q-1}, q \neq 1, q<2
$$

where $f(x)$ is a density function. When $q \rightarrow 1$, the above entropy measure goes to the Shannon entropy measure. By optimizing Mathai's entropy measure, we get a model called pathway model, which consists of many of the standard distributions in statistical literature as special cases see [15].

For the real scalar continuous case the pathway model is represented by the following density function.

$$
g_{1}(x)=c_{1} x^{\nu-1}\left[1-a(1-q) x^{\delta}\right]^{\frac{1}{1-q}}, \delta>0, a>0, \nu>0,
$$


for $0<x<\frac{1}{[a(1-q)]^{\frac{1}{\delta}}}$ and the normalizing constant $c_{1}=\frac{\delta[a(1-q)]^{\frac{\nu}{\delta}} \Gamma\left(\frac{\nu}{\delta}+\frac{1}{1-q}+1\right)}{\Gamma\left(\frac{\nu}{\delta}\right) \Gamma\left(\frac{1}{1-q}+1\right)}$, which is available by optimizing (7) under two general moment-like constraints. For $q<1$ the model remains as a generalized type- 1 beta model in the real case. For $q>1$ we may write $(1-q)=-(q-1)$ so that $g_{1}(x)$ takes the form

$$
g_{2}(x)=c_{2} x^{\nu-1}\left[1+a(q-1) x^{\delta}\right]^{-\frac{1}{q-1}}, x \geq 0, \delta>0, a>0, \nu>1,
$$

which is a generalized type-2 beta model for real $x$ and the normalizing constant $c_{2}=\frac{\delta[a(q-1)]^{\frac{\nu}{\delta}} \Gamma\left(\frac{1}{q-1}\right)}{\Gamma\left(\frac{\nu}{\delta}\right) \Gamma\left(\frac{1}{q-1}-\frac{\nu}{\delta}\right)}, \frac{1}{q-1}-\frac{\nu}{\delta}>$ 0 . For $q>1$, (9) gives the superstatistics of Bect et al. [17, 16]. For $\nu=1, \delta=1$, (9) gives Tsallis statistics [18]. Note that for $q<1$, the density in (9) belongs to the generalized type- 1 beta family and for $q>1$, the density in (10) stays in the generalized type-2 beta family of densities. When $q \rightarrow 1$ the forms in (9) and (10) reduce to the generalized gamma form

$$
g(x)=c x^{\nu-1} \mathrm{e}^{-a x^{\delta}}, x \geq 0,
$$

and $g(x)=0$ elsewhere; and the normalizing constant $c=\frac{\delta a^{\frac{\nu}{\delta}}}{\Gamma\left(\frac{\nu}{\delta}\right)}$. It is not difficult to show that the normalizing constants in (9) and (10) reduce to the normalizing constant in (11). This can been seen by expanding the gamma functions with the help of Stirling's formula, which is given by

$$
\Gamma(z+\alpha) \approx \sqrt{(2 \pi) z^{z+\alpha-\frac{1}{2}}} \mathrm{e}^{-z} \text { for }|z| \rightarrow \infty \text { and } \alpha \text { a bounded quantity. }
$$

The model (11) includes generalized gamma, gamma, exponential, chi-square, Weibull, Maxwell-Boltzmann, Rayleigh distribution, etc. Densities appearing in a number of physical problems are seen to be special cases of (9), a discussion of which may be seen from [14]. For example, $\delta=2, \nu=2, q \rightarrow 1, x>0$ in (10) is the MaxwellBoltzmann density; for $\delta=2, \nu=1, q \rightarrow 1,-\infty<x<\infty$ in (10) is the Gaussian density; for $\nu=\delta, q \rightarrow 1$ is the Weibull density. Here we consider a $q$-analogue of the Nakagami model, which is a special case of Mathai [15], by taking $\delta=2, \nu=2 m$ and $a=\frac{m}{y}$ in (10). Then for $x>0$ and for $q>1, g_{2}(x)$ reduces to the following density which we call $q$-Nakagami density:

$$
f_{1}(x \mid y)= \begin{cases}\frac{2 \Gamma\left(\frac{1}{q-1}\right)(m(q-1))^{m}}{\Gamma(m) \Gamma\left(\frac{1}{q-1}-m\right) y^{m}} x^{2 m-1}\left[1+(q-1) \frac{m x^{2}}{y}\right]^{-\frac{1}{q-1}}, & x \geq 0, m>0, q>1, \nu>0 \\ 0, & \text { otherwise, }\end{cases}
$$

for $\frac{1}{q-1}-m>0, q>1, m>0$. Thus, the parameter $q$ creates a path of going from the type- 1 beta family to type-2 beta family to a gamma family of functions. The movement of $q$ will provide thicker or thinner tailed distributions which will also be helpful in a modeling situation where one may be looking for a thicker or thinner tailed distribution as appropriate fit.

\section{Extension of Nakagami-gamma distribution}

Let us replace $p_{1}(x \mid y)$ by a pathway model $f_{1}(x \mid y)$ in (12), then the density function of the received power $\gamma$ denoted by $h_{1}(x)$ has the following form

$$
\begin{aligned}
h_{1}(x) & =\int_{y} f_{1}(x \mid y) p_{2}(y) \mathrm{d} y \\
& =\frac{2 \Gamma\left(\frac{1}{q-1}\right)(m(q-1))^{m} \delta^{\delta}}{\Gamma(m) \Gamma(\delta) \Gamma\left(\frac{1}{q-1}-m\right) \beta^{\delta}} x^{2 m-1} \int_{0}^{\infty} y^{\delta-m-1} \mathrm{e}^{-\frac{\delta y}{\beta}}\left[1+(q-1) \frac{m x^{2}}{y}\right]^{-\frac{1}{q-1}} \mathrm{~d} y,
\end{aligned}
$$

for $q>1, \frac{1}{q-1}-m>0, m>0, \delta>0$. The integral part in (13) can be evaluated by using Mellin convolution property, by taking it as a statistical distribution problem. Let $x_{1}$ and $x_{2}$ be independently distributed real scalar positive random variables. Let the densities be $h_{3}\left(x_{1}\right)$ and $h_{4}\left(x_{2}\right)$ respectively. Consider the transformation 
$u=x_{1} x_{2}$ and $v=x_{1}$. Then the density of $u$ is given by

$$
g(u)=\int_{v} \frac{1}{v} h_{3}(v) h_{4}\left(\frac{u}{v}\right) \mathrm{d} v
$$

Let

$$
h_{3}\left(x_{1}\right)=\mathrm{c}_{3} x_{1}^{\delta-m} \mathrm{e}^{-\frac{\delta x_{1}}{\beta}}, x_{1}>0, \delta, m, \beta>0
$$

and

$$
h_{4}\left(x_{2}\right)=\mathrm{c}_{4}\left[1+(q-1) x_{2}\right]^{-\frac{1}{q-1}}, x_{2}>0, q>1,
$$

so that $h_{3}\left(x_{1}\right)$ and $h_{4}\left(x_{2}\right)$ can create statistical densities for appropriate values of the normalizing constants $c_{3}$ and $c_{4}$. Therefore

$$
c_{3} c_{4} \int_{y=0}^{\infty} \frac{1}{y} y^{\delta-m} \mathrm{e}^{-\frac{\delta y}{\beta}}\left[1+(q-1) \frac{m x^{2}}{y}\right] \mathrm{d} y=\int_{0}^{\infty} \frac{1}{y} h_{1}(y) h_{2}\left(\frac{m x^{2}}{y}\right) \mathrm{d} y .
$$

Hence the Mellin transform of the left side is the product of the Mellin transform of the right side. Writing in terms of statistical expectations,

$$
E\left(x_{1}{ }^{s-1}\right)=c_{3}\left(\frac{\beta}{\delta}\right)^{s+\delta-m} \Gamma(s+\delta-m), \Re(s+\delta-m)>0, \beta, \delta>0,
$$

where $\Re($.$) denotes the real part of (.), and$

$$
E\left(x_{2}{ }^{s-1}\right)=c_{4}\left(\frac{1}{q-1}\right)^{s} \frac{\Gamma(s) \Gamma\left(\frac{1}{q-1}-s\right)}{\Gamma\left(\frac{1}{q-1}\right)}, \Re(s)>0, \Re\left(\frac{1}{q-1}-s\right)>0 .
$$

From (16) and (17),

$$
E\left(u^{s-1}\right)=c_{3} c_{4}\left(\frac{\beta}{\delta}\right)^{s+\delta-m}\left(\frac{1}{q-1}\right)^{s} \frac{\Gamma(s+\delta-m) \Gamma(s) \Gamma\left(\frac{1}{q-1}-s\right)}{\Gamma\left(\frac{1}{q-1}\right)},
$$

for $\Re(s)>0, \Re(s+\delta-m)>0, \Re\left(\frac{1}{q-1}-s\right)>0$. For inverting the moment expression to get the density we need the moments for complex variable $s$. Hence we have taken arbitrary moments. Then the integral is available by taking the inverse Mellin transform and it is the following:

$$
\begin{gathered}
c_{3} c_{4} \int_{y=0}^{\infty} \frac{1}{y} y^{\delta-m} \mathrm{e}^{-\frac{\delta y}{\beta}}\left[1+(q-1) \frac{m x^{2}}{y}\right]^{-\frac{1}{q-1}} \mathrm{~d} y, i=\sqrt{-1} \\
=c_{3} c_{4} \frac{1}{\Gamma\left(\frac{1}{q-1}\right)}\left(\frac{\beta}{\delta}\right)^{\delta-m} \frac{1}{2 \pi i} \int_{L} \Gamma(\delta-m+s) \Gamma(s) \Gamma\left(\frac{1}{q-1}-s\right)\left(\frac{m \delta(q-1) x^{2}}{\beta}\right)^{-s} \mathrm{~d} s \\
=c_{3} c_{4} \frac{1}{\Gamma\left(\frac{1}{q-1}\right)}\left(\frac{\beta}{\delta}\right)^{\delta-m} \mathrm{G}_{1,2}^{2,1}\left[\left.\frac{\delta(q-1) m x^{2}}{\beta}\right|_{0, \delta-m} ^{1-\frac{1}{q-1}}\right], x \geq 0,
\end{gathered}
$$

for $q>1, \beta, m, \delta>0$, and $h_{1}(x)=0$ elsewhere, where $G$ is the $G$-function. For the definition, theory, applications and computable representations of $G$-function see [19]. From (19) we have the density of $x$, denoted by $h_{1}(x)$, given by the following:

$$
h_{1}(x)=\frac{2(m(q-1))^{m}}{\Gamma(m) \Gamma(\delta) \Gamma\left(\frac{1}{q-1}-m\right)}\left(\frac{\delta}{\beta}\right)^{m} x^{2 m-1} \mathrm{G}_{1,2}^{2,1}\left[\left.\frac{\delta(q-1) m x^{2}}{\beta}\right|_{0, \delta-m} ^{1-\frac{1}{q-1}}\right],
$$


for $x \geq 0, \frac{1}{q-1}-m>0, m, \delta>0$, and $h_{1}(x)=0$ elsewhere. Using the definition of Whittaker function, $h_{1}(x)$ becomes

$$
\begin{aligned}
h_{1}(x) & =\frac{2(m(q-1))^{m} \Gamma\left(\frac{1}{q-1}\right) \Gamma\left(\frac{1}{q-1}+\delta-m\right)}{\beta^{m} \Gamma(m) \Gamma(\delta) \Gamma\left(\frac{1}{q-1}-m\right)}\left(\frac{\delta(q-1) m x^{2}}{\beta}\right)^{\frac{\delta-m-1}{2}} \mathrm{e}^{\frac{\delta(q-1) m x^{2}}{2 \beta}} \\
& \times \mathrm{W}_{\frac{m}{2}-\frac{1}{q-1}-\frac{\delta}{2}+\frac{1}{2}, \frac{m-\delta}{2}}\left(\frac{\delta(q-1) m x^{2}}{\beta}\right),
\end{aligned}
$$

for $x \geq 0, \frac{1}{q-1}-m>0, \frac{1}{q-1}+\delta-m>0, m, \beta, \delta>0$ and $h_{1}(x)=0$ elsewhere. The reason for the choice of terminology is that this new extension of the gamma distribution can describe impulsive data and its density function has a thicker tail when compared to the classical gamma distribution.

\subsection{Properties of extended Nakagami-gamma distribution}

\section{Theorem 1}

When the density function of the received power $\gamma$ conditioned on the average power $y$ is of the form $f_{1}(x \mid y)$ in (12), when the density function of the average power due to shadowing is of the form $p_{2}(y)$ in $(3)$ then the unconditional (received signal power due to both multipath and shadowing) density of $x$, denoted by $h_{1}(x)$ is given by

$$
\begin{aligned}
h_{1}(x) & =\frac{2(m(q-1))^{m} \Gamma\left(\frac{1}{q-1}\right) \Gamma\left(\frac{1}{q-1}+\delta-m\right)}{\beta^{m} \Gamma(m) \Gamma(\delta) \Gamma\left(\frac{1}{q-1}-m\right)}\left(\frac{\delta(q-1) m x^{2}}{\beta}\right)^{\frac{\delta-m-1}{2}} \mathrm{e}^{\frac{\delta(q-1) m x^{2}}{2 \beta}} \\
& \times \mathrm{W}_{\frac{m}{2}-\frac{1}{q-1}-\frac{\delta}{2}+\frac{1}{2}, \frac{m-\delta}{2}}\left(\frac{\delta(q-1) m x^{2}}{\beta}\right),
\end{aligned}
$$

for $x \geq 0, \frac{1}{q-1}-m>0, \frac{1}{q-1}+\delta-m>0, m, \beta, \delta>0$ and $h_{1}(x)=0$ elsewhere. As $q \rightarrow 1, h_{1}(x)$ tends to $h(x)$ where $h(x)$ is the Nakagami-gamma density with parameters $\beta, \delta, q$ and $m$, and it is given by

$$
\left.h(x)=\frac{4}{\Gamma(m) \Gamma(\delta)}\left(\frac{m \delta}{\beta}\right)^{\frac{m+\delta}{2}} x^{m+\delta-1} K_{\delta-m}\left(2 \sqrt{(} \frac{\delta m}{\beta}\right) x\right),
$$

for $x \geq 0, \beta, m, \delta>0$ and $h(x)=0$ elsewhere.

In the following section, we discuss the statistical properties of the extended Nakagami-gamma model.

\subsection{Behavior of extended Nakagami-gamma model}

The possible shapes of $h_{1}(x)$ in (22) are provided for some selected values of the parameters in Figure 1. Figure 1, shows the density curves for the extended Nakagami-gamma density for selected values of the parameters $m, \beta, \delta$ and for various values of the parameter $q$. In Figure 1, we take $m=2, \delta=3, \beta=3$ and allow $q$ to vary from 1 and 2 so that one can obtain thicker tailed curves for the same set of $m=2, \delta=3, \beta=3$ by varying $q$ for $1<q<2$. The main advantage of the extended model is that one can see the movement of the basic density, producing thicker tails as pathway parameter $q$ varies.

\subsection{Moments}

The $h^{\text {th }}$ moment of a random variable $x$ with the density function (22) is

$$
\begin{aligned}
E\left(x^{h}\right) & =\int_{0}^{\infty} x^{h} h_{1}(x) \mathrm{d} x \\
& =\left(\frac{\delta(q-1) m}{\beta}\right)^{-\frac{h}{2}} \frac{\Gamma\left(m+\frac{h}{2}\right) \Gamma\left(\delta+\frac{h}{2}\right) \Gamma\left(\frac{1}{q-1}-m-\frac{h}{2}\right)}{\Gamma(m) \Gamma(\delta) \Gamma\left(\frac{1}{q-1}-m\right)}
\end{aligned}
$$

for $q>1, m>0, \delta>0, \beta>0, \frac{1}{q-1}-m>0, \Re\left(\frac{1}{q-1}-m-\frac{h}{2}\right)>0, \Re\left(\delta+\frac{h}{2}\right)>0, \Re\left(m+\frac{h}{2}\right)>0$. 


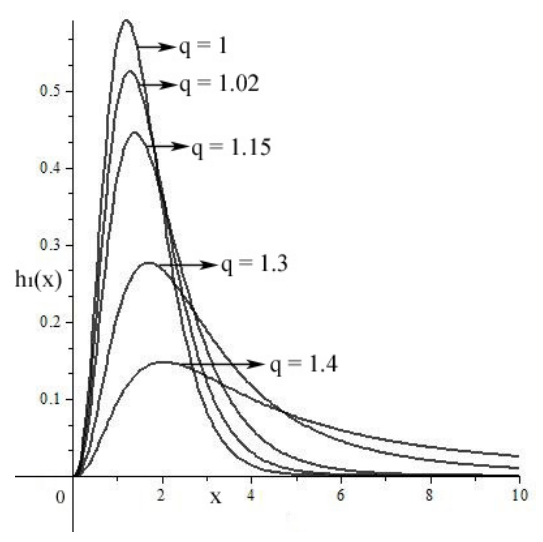

Figure 1. Density functions of extended Nakagami-gamma density: $\beta=3, m=2, \delta=3$ fixed, and for different values of $q$.

\subsection{Distribution function}

The distribution function $H_{1}(x)$ of the extended Nakagami-gamma density function $h_{1}(x)$ is given by

$$
H_{1}(x)=1-\frac{1}{\Gamma(m) \Gamma(\delta) \Gamma\left(\frac{1}{q-1}-m\right)}\left(\frac{\beta}{m \delta(q-1)}\right) H_{2,3}^{3,1}\left[\left.\left(\frac{\delta(q-1) m}{\beta}\right)^{\frac{1}{2}} x\right|_{(0,1),\left(m, \frac{1}{2}\right),\left(\delta, \frac{1}{2}\right)} ^{\left(1+m-\frac{1}{q-1}+\frac{1}{2}, \frac{1}{2}\right),(1,1)}\right],
$$

for $x \geq 0, q>1, m, \delta, \beta>0, \frac{1}{q-1}-m>0$, where $H_{2,3}^{3,1}$ is a $H$-function, which is a generalization of the $G$ function. For theory and applications of $H$-function see [20]. Computable representation of this function is also available in the literature.

\subsection{Laplace transform}

The Laplace transform $L_{h_{1}}(t)$ of the density function $h_{1}(x)$ is given by

$$
L_{h_{1}}(t)=\int_{0}^{\infty} \mathrm{e}^{-t x} h_{1}(x) \mathrm{d} x .
$$

Using equation (2.19) in Mathai et al. (2010), we get

$$
L_{h_{1}}(t)=\frac{(m \delta(q-1))^{\frac{1}{2}}}{t \Gamma(m) \Gamma(\delta) \Gamma\left(\frac{1}{q-1}-m\right) \beta^{\frac{1}{2}}} \mathrm{H}_{2,2}^{2,2}\left[\left.\left(\frac{\delta(q-1) m}{\beta}\right)^{\frac{1}{2}} \frac{1}{t}\right|_{\left(m-\frac{1}{2}, \frac{1}{2}\right),\left(\delta-\frac{1}{2}, \frac{1}{2}\right)} ^{(0,1),\left(\frac{1}{2}+m-\frac{1}{q-1}, \frac{1}{2}\right)}\right],
$$

for $t>0, m>0, \delta>0, \beta>0, q>1, \frac{1}{q-1}-m>0$.

\section{Statistics of the output SNR}

Let us consider a single receiver system operating over the extended Nakagami-gamma composite fading channel in the presence of Additive White Gaussian Noise (AWGN). The performance of any communication system, in terms of Bit Error Rate (BER) and signal outage will depend on the instantaneous SNR per received signal is given by

$$
\gamma=x^{2} \frac{E_{s}}{N_{0}},
$$

where $E_{s}$ is the average transmitted signal per energy and $N_{0}$ is the single sided AWGN power spectral density. The corresponding average SNR is

$$
\bar{\gamma}=E\left(x^{2}\right) \frac{E_{s}}{N_{0}},
$$


where $E($.$) denotes the expectation and$

$$
\bar{\gamma}=\int_{0}^{\infty} \gamma p_{\gamma}(\gamma) \mathrm{d} \gamma
$$

is the average SNR. In this section, closed-form expressions for the moments of the output SNR of single receiver system operating over extended Nakagami-gamma fading channels, are derived. Here we will compute the density of $\gamma$ in (28) by using the density of $x$ in (20). The results are given in the form of theorems:

Theorem 2

(Density function): The density function of the instantaneous received SNR can be obtained as

$$
p_{\gamma}(\gamma)=\frac{(\delta m(q-1) d)^{m}}{(\bar{\gamma} \beta)^{m} \Gamma(m) \Gamma(\delta) \Gamma\left(\frac{1}{q-1}-m\right)} \gamma^{m-1} \mathrm{G}_{1,2}^{2,1}\left[\left.\frac{\delta(q-1) m d}{\beta \bar{\gamma}} \gamma\right|_{0, \delta-m} ^{1-\frac{1}{q-1}}\right],
$$

for $\gamma \geq 0, \delta, \beta, m, \bar{\gamma}>0, \frac{1}{q-1}-m>0, q>1$, where $d=\frac{\beta}{1-(m+1)(q-1)}$ and $p_{\gamma}(\gamma)=0$ elsewhere.

Theorem 3

(Moments): The $h^{\text {th }}$ moment of $\gamma$ can be found in closed form as

$$
E\left(\gamma^{h}\right)=\left(\frac{\beta \bar{\gamma}}{\delta(q-1) m d}\right)^{h} \frac{\Gamma(\delta+h) \Gamma(m+h) \Gamma\left(\frac{1}{q-1}-m-h\right)}{\Gamma(\delta) \Gamma(m) \Gamma\left(\frac{1}{q-1}-m\right)},
$$

for $\beta, \delta, m, d>0, \bar{\gamma}>0, \frac{1}{q-1}-m>0, \Re\left(\frac{1}{q-1}-m-h\right)>0, \Re(\delta+h)>0, \Re(m+h)>0$.

Theorem 4

(Distribution function): The distribution function of SNR is given by

$$
P_{\gamma}(\gamma)=1-\frac{1}{\Gamma(m) \Gamma(\delta) \Gamma\left(\frac{1}{q-1}-m\right)} \mathrm{G}_{2,3}^{3,1}\left[\left.\frac{\delta(q-1) m d}{\beta \bar{\gamma}} \gamma\right|_{0, m, \delta} ^{1,1+m-\frac{1}{q-1}}\right],
$$

for $\gamma>0, \frac{1}{q-1}-m>0, m, \delta, \beta, d>0, \bar{\gamma}>0, q>1$.

Theorem 5

(Laplace transform): The Laplace transform of the instantaneous SNR is given by

$$
L_{p_{\gamma}}(t)=\frac{m(q-1) \delta d}{t \beta \bar{\gamma} \Gamma(m) \Gamma(\delta) \Gamma\left(\frac{1}{q-1}-m\right)} \mathrm{G}_{2,2}^{2,2}\left[\left.\frac{\delta(q-1) m d}{\beta \bar{\gamma} t}\right|_{m-1, \delta-1} ^{0, m-\frac{1}{q-1}}\right],
$$

for $m, d, \delta, \beta>0, \frac{1}{q-1}-m>0, \bar{\gamma}>0, t>0$.

\subsection{Performance analysis}

In this section, we derive the expressions of the Amount of Fading (AF), Outage Probability (OP), Average channel Capacity (AC), Outage Capacity (OC) and Average Bit Error Probability (ABEP) for digital communication system operating over extended Nakagami-gamma fading channel, using the expressions given in theorems 1, 2, 3, 4 .

\subsection{Amount of Fading (AF)}

The AF is introduced by Simon and Alouini [1] as a unified performance measure of the severity of fading, is an important measure for the performance of a diversity system since it can be utilized to parameterize the distribution of the signal to noise ration (SNR) of the received signal. The amount of fading is defined as the ratio of the variance to the square average of the instantaneous SNR. That is

$$
A F=\frac{E\left(\gamma^{2}\right)}{[E(\gamma)]^{2}}-1
$$


Then using (31), the AF can be easily derived as

$$
A F=\frac{\Gamma(\delta+2) \Gamma(m+2) \Gamma\left(\frac{1}{q-1}-m-2\right) \Gamma(\delta) \Gamma(m) \Gamma\left(\frac{1}{q-1}-m\right)}{\Gamma^{2}(\delta+1) \Gamma^{2}(m+1) \Gamma^{2}\left(\frac{1}{q-1}-m-1\right)}-1,
$$

for $\delta, m>0, \frac{1}{q-1}-m-2>0, \frac{1}{q-1}-m-1>0, \frac{1}{q-1}-m>0$. It can be seen that the AF depends on fading parameter $m$ and shadowing parameter $\delta$.

\subsection{Outage Probability Performance (OP)}

The outage probability is defined as the probability that the instantaneous error rate exceeds a specified value or equivalently, the probability that the output signal to noise ratio(SNR), $\gamma$, falls below a predetermined threshold $\gamma_{0}$ (i.e, $\gamma<\gamma_{0}$ ),

$$
P_{\text {out }}=P\left(0 \leq \gamma \leq \gamma_{0}\right)=\int_{0}^{\gamma_{0}} p_{\gamma}(\gamma) \mathrm{d} \gamma
$$

where $p_{\gamma}(\gamma)$ is the density function of $\gamma$ obtained in (30). In other words, $P_{\text {out }}$ is the cumulative distribution function (CDF) of the random variable $\gamma$ evaluated at $\gamma_{0}$. Then $P_{\text {out }}$ is directly given by

$$
P_{\text {out }}=P_{\gamma}\left(\gamma_{0}\right)
$$

where $P_{\gamma}(\gamma)$ is given in (32).

In Figure 2, the $P_{\text {out }}$ is plotted as a function of the normalized outage threshold, $\frac{\gamma_{0}}{\bar{\gamma}}$, for several values of $m$ and $\delta$.

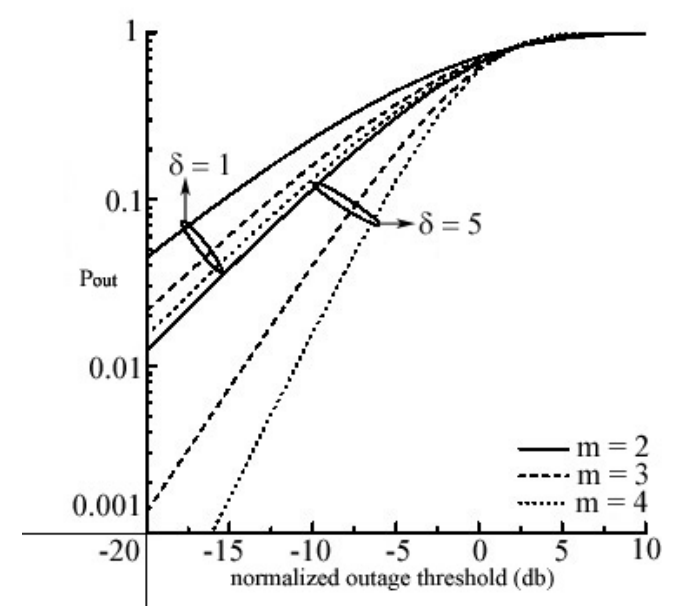

Figure 2. Outage probability versus the normalized outage threshold for several values of $m$ and $\delta$.

\subsection{Average Channel Capacity (AC)}

Based on the Shannon's theorem, the average channel capacity $C$ can be calculated by averaging the instantaneous channel capacity over SNR as

$$
\bar{C}_{\gamma}=W \int_{0}^{\infty} \ln (1+\gamma) p_{\gamma}(\gamma) \mathrm{d} \gamma
$$

where $W$ is the signal transmission bandwidth and $p_{\gamma}(\gamma)$ is given in (30). Using the definition of $G$-function, we get

$$
\bar{C}_{\gamma}=\frac{W(\delta(q-1) m d)^{m}}{(\bar{\gamma} \beta)^{m} \Gamma(m) \Gamma(\delta) \Gamma\left(\frac{1}{q-1}-m\right)} \times I_{1},
$$


where

$$
I_{1}=\int_{0}^{\infty} \gamma^{m-1} \mathrm{G}_{2,2}^{1,2}\left[\left.\gamma\right|_{1,0} ^{1,1}\right] \mathrm{G}_{1,2}^{2,1}\left[\left.\frac{\delta(q-1) m d}{\beta \bar{\gamma}} \gamma\right|_{0, \delta-m} ^{1-\frac{1}{q-1}}\right] \mathrm{d} \gamma
$$

Using the Mellin transform of the product of two $G$-functions, see [20], we get

$$
\bar{C}_{\gamma}=\frac{W}{\Gamma(m) \Gamma(\delta) \Gamma\left(\frac{1}{q-1}-m\right)} G_{4,3}^{2,4}\left[\left.\frac{\beta \bar{\gamma}}{\delta(q-1) m d}\right|_{1, \frac{1}{q-1}-m, 0} ^{1,1,1-m, 1-\delta}\right],
$$

for $m, \delta, \beta, \bar{\gamma}, d>0, q>1, \frac{1}{q-1}-m>0$.

\subsection{Outage Capacity (OC)}

The outage capacity is another important statistical measure to quantify the spectral efficiency in fading channels, which is defined as the probability that the instantaneous capacity, $C_{\gamma}$ falls below a certain specified threshold $C_{0}$ i.e,

$$
C_{\text {out }}=P\left(0 \leq C_{\gamma} \leq C_{0}\right)=\int_{0}^{C_{0}} p_{C_{\gamma}(c)} \mathrm{d} c
$$

where $p_{C_{\gamma}}(c)$ is the density function of the instantaneous capacity $C_{\gamma}$, defined in (37). The outage capacity (OC), $C_{\text {out }}$ can be given in terms of the cumulative distribution function of the channel capacity as follows

$$
C_{\text {out }}=P_{\gamma}\left(2^{C_{0} \mid W}-1\right) \text {. }
$$

Then the outage capacity of the extended Nakagami-gamma composite fading channel as follows,

$$
C_{\text {out }}=1-\frac{1}{\Gamma(m) \Gamma(\delta) \Gamma\left(\frac{1}{q-1}-m\right)} \mathrm{G}_{2,3}^{3,1}\left[\left.\frac{\delta(q-1) m d}{\beta \bar{\gamma}}\left(2^{C_{0} \mid W}-1\right)\right|_{0, m, \delta} ^{1,1+m-\frac{1}{q-1}}\right],
$$

for $m, \delta, \beta, d>0, \frac{1}{q-1}-m>0, \bar{\gamma}>0$.

\subsection{Average Bit Error Probability (ABEP)}

The instantaneous bit error probabilities (IBEP), conditioned on the instantaneous SNR $\gamma$, in an AWGN channel can be written in compact form as

$$
P_{E}(\gamma)=\frac{\Gamma(c, a \gamma)}{2 \Gamma(c)}
$$

where $\Gamma(.,$.$) is the incomplete gamma function. The parameter a$ and $b$ take specific values for specific modulations. For instance $a=\frac{1}{2}$ for coherent detection and 1 for noncoherent/differentially coherent detection, and $c=\frac{1}{2}$ for orthogonal FSK and 1 for antipodal PSK. Using $p_{\gamma}(\gamma)$, the average BEP is given by

$$
\overline{P_{E}}(\gamma)=\int_{0}^{\infty} P_{E}(\gamma) p_{\gamma}(\gamma) \mathrm{d} \gamma
$$

Then substituting (43) and $P_{E}(\gamma)$ in to (44), the ABEP in extended Nakagami-gamma fading channel is given in closed form in the following theorem.

\section{Theorem 6}

(Average Bit Error Probability): Let $\gamma$, be the instantaneous SNR at the output of the single receiver operating in extended Nakagami-gamma wireless fading channels. Then the ABEP $\overline{P_{E}}$ is given by

$$
\overline{P_{E}}(\gamma)=\frac{1}{\Gamma(m) \Gamma(\delta) \Gamma\left(\frac{1}{q-1}-m\right) \Gamma(c)} G_{3,3}^{3,2}\left[\left.\frac{\beta \bar{\gamma}}{\delta(q-1) m d}\right|_{c, \frac{1}{q-1}-m, 0} ^{1-m, 1-\delta, 1}\right],
$$

for $\beta, \delta, m, c, d>0, \frac{1}{q-1}-m>0$. 
Proof: Substituting (30) and $P_{E}(\gamma)$ in to (44), the ABEP $\overline{P_{E}}(\gamma)$ can be given as

$$
\bar{P}_{E}(\gamma)=\frac{(\delta m(q-1) d)^{-m}}{\Gamma(m) \Gamma(\delta) \Gamma\left(\frac{1}{q-1}-m\right) \Gamma(c)(\bar{\gamma} \beta)^{-m}} \int_{0}^{\infty} \Gamma(c, a \gamma) \gamma^{m-1} \mathrm{G}_{1,2}^{2,1}\left[\left.\frac{\delta(q-1) m d}{\beta \bar{\gamma}}\right|_{0, \delta-m} ^{1-\frac{1}{q-1}}\right]
$$

substituting Meijer's $G$ function representation of both the incomplete gamma and $p_{g}(\gamma)$, we get

$$
{\overline{P_{E}}}_{(\gamma)}=\frac{(\delta m(q-1) d)^{-m}}{\left.\Gamma(m) \Gamma(\delta) \Gamma\left(\frac{1}{q-1}-m\right) \Gamma(c)(\overline{(\gamma}) \beta\right)^{-m}} \times I_{2},
$$

where

$$
I_{2}=\int_{0}^{\infty} \gamma^{m-1} \mathrm{G}_{1,2}^{2,0}\left[\left.a \gamma\right|_{c, 0} ^{1}\right] \mathrm{G}_{1,2}^{2,1}\left[\left.\frac{\delta(q-1) m d}{\beta \bar{\gamma}} \gamma\right|_{0, \delta-m} ^{1-\frac{1}{q-1}}\right] \mathrm{d} \sigma
$$

Using the Mellin transform of the product of two $G$-functions, see Mathai et al. (2010), we get

$$
\overline{P_{E}}(\gamma)=\frac{1}{\Gamma(m) \Gamma(\delta) \Gamma(c) \Gamma\left(\frac{1}{q-1}-m\right)} \mathrm{G}_{3,3}^{3,2}\left[\left.\frac{\beta \bar{\gamma}}{\delta(q-1) m d}\right|_{c, \frac{1}{q-1}-m, 0} ^{1-m, 1-\delta, 1}\right],
$$

for $\beta, \delta, m, d>0, \frac{1}{q-1}-m>0, \bar{\gamma}>0, q>1$.

The performance of ABEP for the coherent BPSK are seen in the following figure.

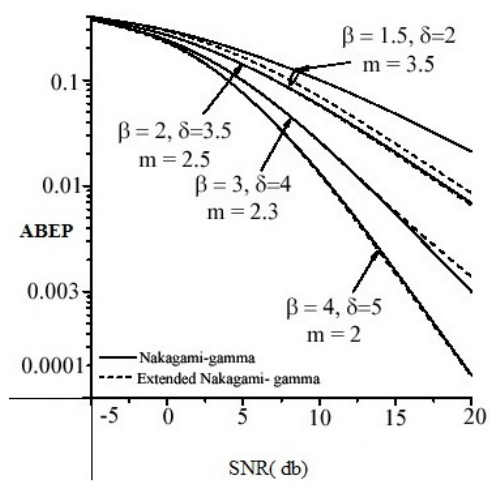

Figure 3. The ABEP of Nakagamai-gamma and extended Nakagami-gamma fading channels versus the average input SNR for several values of $m, \beta$ and $\delta$.

\section{Conclusion}

In this paper, we have developed an extended form of a statistical model for composite fading channel modeling. The density function is evaluated in terms of $G$-function. In addition, the distribution function, the Laplace transform and moments of these random variables are determined. These derived results can be used to analyze the outage probability and the bit error probability in digital communication systems operating over multipath and shadow fading channels.

\section{Acknowledgement}

The author would like to thank Professor A. M. Mathai of the Centre for Mathematical Sciences and Professor S.K. Singh of Banaras Hindu University for fruitful discussions. The author would like to thank the Department 
of Science and Technology and UGC, Government of India, New Delhi, for the financial assistance for this work under project-number SR/S4/MS:287/05, and the Centre for Mathematical Sciences for providing all facilities.

\section{REFERENCES}

1. M. K. Simon, and M. S. Alouini, Digital Communication Over Fading Channels, second edition, John Wiely and sons, 2006.

2. M. H. Ismail, and M. M. Matalgah, Performance of dual maximal ratio combining diversity in non-identical correlated Weibull fading channels using Padé approximation, IEEE Transactions on Communications, vol. 54, pp. 397-402, 2006.

3. Tjeng T. Tjhung, Fade Statistics in Nakagami-Lognormal Channels, IEEE Transactions on Communications, vol. 47, pp. 1769$1772,1999$.

4. H. Suzuki, A statistical model for urban radio propagation, IEEE Transactions on Communications, vol. 25, pp. 673-680, 1977.

5. P. Bithas, Weibull-Gamma composite distribution: An alternative multipath/shadowing fading model, Electronics Letters, vol. 45, pp. 749-751, 2009.

6. A. Abdi, and M. Kaveh, Comparison of DPSK and MSK bit error rates for K and Rayleigh-lognormal fading distributions, IEEE Communications Letters, vol. 4, pp.122-124, 2000.

7. P. M. Shankar, Error rates in generalized shadowed fading channels, Wireless Personal Communications, vol. 28, pp. 233-238, 2004.

8. S. Nadarajah, and S. Kotz, Compound statistical models for shadowing fading channels, International Journal of Electronics and Communications, vol. 62, pp. 138-142, 2008.

9. S. Atapattu, C. Tellambura, and H. Jiang, A mixture gamma distribution to model the SNR of wireless channels, IEEE transactions on wireless communications, vol. 10, pp. 4193-4203, 2011.

10. P. M. Shankar, Outage analysis in wireless channels with multiple interferers subject to shadowing and fading using a compound pdf model, International Journal of Electronics and Communications, vol. 61, pp. 255-261, 2007.

11. E. T. Jaynes, Probability Theory: The Logic of Science, Cambridge University Press, 2003.

12. M. Debbah, and R. R. Müller, MIMO channel modelling and the prinicple of maximum entropy, IEEE Transations on Information Theory, vol. 15, pp. 1667-1690, 2005.

13. M. Guillaud, M. Debbah and A. U. Moustakas, A maximum entropy characterization of spatially correlated MIMO wirelesss channels, IEEE Wireless communications and Networking Conference, Hong Kong, 2007.

14. A. M. Mathai, and H. J. Haubold, Pathway model, superstatistics Tsallis statistics and a generalized measure of entropy, Physica A, vol. 375, pp. 110-122, 2007.

15. A. M. Mathai, A pathway to matrix - variate gamma and normal densities, Linear Algebra and Its Applications, vol. 396, pp. $317-328,2005$

16. C. Beck, Stretched exponentials from superstatistics, Physica A, vol. 365, pp. 96-101, 2006.

17. C. Beck, and E. G. D. Cohen, Superstatistcs, Physica A, vol. 322, pp. 267-275, 2003.

18. C. Tsallis, What should a statistical mechanics satisfy to reflect nature? Physica D, vol. 193, pp. 3-34, 2004.

19. A. M. Mathai, A Handbook of Generalized Special Functions for Statistical and Physical Sciences, Clarendon Press, Oxford, 1993.

20. A. M. Mathai, R. K. Saxena, and H. J. Haubold, The H-function: Theory and Applications, Springer, New York, 2010. 